\title{
When the environmental fate of chemicals is affected by the formation of metastable states: quantification of sorption-desorption hysteresis

\author{
MIKHAIL BORISOVER
}

The Institute of Soil, Water and Environmental Sciences, The Volcani Center

Presenting Author: vwmichel@volcani.agri.gov.il

The biogeochemical fate of pollutants in various environments, including soils and sediments, is often controlled by sorption-desorption hysteresis (SDH). The SDH affects concentrations of chemicals in environmental phases, their release and bioavailability. When ruling out sorbate degradation, covalent binding, non-accounted losses of competitors and suspended matter, or overlooked slow kinetics, one mechanism responsible for SDH was related in earlier literature to sorbent modification caused by sorbate-sorbent interactions [1,2]. Metastable states formed across sorption remain non-relaxed during desorption, causing SDH but allowing free exchange of sorbate and solvent molecules between phases. For such kind of hysteresis, this paper represents a new approach quantifying the SDH extent in terms of free energy contribution caused by nonrelaxed changes in a sorbed state $[3,4]$. The quantification is reached by a proper integration along a sequence including sorption isotherm (SI) and successive desorption isotherm (DI). A simple result is obtained when the isotherms follow the common Freundlich models. In that case, extra-free energy (normalized by RT; R and T are the universal gas constant and absolute temperature, respectively) accumulated in a state across DI as compared to the same composition state of SI is given as $(1 / m-1 / n)$ where $n$ and $m$ are the Freundlich exponents of sorption and successive desorption branches, respectively. The whole approach is illustrated by considering the data when DI is a sequence of multiple steps or obtained as a so-called "one-point DI". Using the available experimental data on SDH for hydrocarbons and pesticides interacting with soils, minerals, sediments and artificial sorbent materials, excessive free energies associated with SDH are obtained and discussed in terms of effects of (i) sorbate structure, (ii) solute concentrations, and (iii) nature of sorbents. The proposed approach contributes to our better understanding of the biogeochemical fate of chemicals when SDH is supposed to have a thermodynamic justification.

[1] Sander, M., Lu, Y., \& Pignatello, J.J. (2005), Journal of Environmental Quality, 34, 1063-1072.

[2] Sander, M., \& Pignatello, J.J. (2009), Environmental Toxicology \& Chemistry 28, 447-457.

[3] Borisover, M. (2019), Chemosphere 215, 490-499.

[4] Borisover, M. (2020), European Journal of Soil Science DOI: $10.1111 /$ ejss. 13043. 